\title{
Analysis of melatonin and serotonin from cherries: a review
}

\author{
Éva Varga-Visi*, Ildikó Jócsák, Katalin Lóki, György Végvári \\ Faculty of Agriculture and Environmental Sciences, Kaposvár University \\ Guba S. u. 40., H-7400 Kaposvár, Hungary
}

\begin{abstract}
The proper quantification of serotonin and melatonin in different foods is indispensable to evaluate the significance of their dietary intake on blood and plasma levels in humans. This review summarizes the issues regarding the measurements of these compounds from sour cherry and sweet cherry. Sample preparation and analytical methods are discussed, mainly focusing on the liquid chromatographic determination of these compounds, with special respect to their parallel determination in one run.
\end{abstract}

Keywords: indoleamine derivatives, LC-MS, electrochemical detection, fluorometric detection

\section{INTRODUCTION}

The high ingestion pattern of sweet cherries has been reported to be health promoting, including the alleviation of arthritic pain, reducing the risk of cancer and neurodegenerative diseases. These multiple health benefits are significantly linked to the strong antioxidant effect of the fruit. Sweet cherries are good sources of many phytochemicals, including anthocyanin flavonoids and other compounds such as phenolic acids. The total antioxidant activity highly correlates to the concentration of these compounds (Ballistreri et al., 2013; Chockchaisawasdee et al., 2016; Wani et al., 2014; Nawirska-Olszańska et al., 2017).

Melatonin, primarily known as a neurohormon in mammals, also occurs in sweet cherries as a phytohormone; parallel with serotonin, its precursor that has a neurotransmitter role in mammals. Moreover, melatonin possesses antioxidant properties, therefore it can contribute to the antioxidant capacity of the fruit. These indoleamine derivatives can be originated from food besides endogenous formation in human as they can be absorbed and enter the bloodstream. The level of dietary intake may have an influence on their levels in blood and tissues and may contribute to their physiological effects, nevertheless, the degree of this contribution is still not completely elucidated (Garcia-Parrilla et al., 2009, González-Gómez \& Lozano, 2009; Feng et al., 2014;

\footnotetext{
*CORRESPONDING AUTHOR

Kaposvár University, Faculty of Agricultural and Environmental Sciences

Institute of Physiology, Biochemistry and Animal Health, Department of Biochemistry

H-7400 Kaposvár, Guba S. str. 40., Tel.: +36-82-505-800

E-mail: vargane.eva@ke.hu
} 
Kirakosyan et al., 2009). Moreover, there is scarce information available in the literature on the melatonin and serotonin content of fruits, especially in the case of sweet cherries. One of the factors that render these studies complicated is that the quantification of indoleamine derivatives is difficult from food matrices. The aim of this brief outline is to summarise the possibilities of the analysis of serotonin and melatonin from cherries.

\section{Sample preparation}

Fruit tissues are usually freeze-dried prior to extraction as it was applied to different cherry cultivars and their products (Burkhardt et al., 2001, GonzálezGómez \& Lozano, 2009; Kirakosyan et al., 2009; Kocadağli et al., 2014; Rosado et al., 2017) and also for several commonly consumed fruits (Huang \& Mazza, 2011). Contrary, some authors (Homoki et al., 2015; Islam et al., 2016, Reinholds et al., 2016) used freshly crushed fruits to extract indoleamines.

There are large differences among the extraction solvents and techniques that are used to obtain indoleamine derivatives (Table 1.) with one exception, i.e. all of the authors carried out extraction under dim light in order to avoid the degradation of analytes. As indole ring is sensitive to light, exposure to UV light can completely destroy this compound in standard solutions (Huang \& Mazza, 2011). Serotonin and melatonin have amphipathic characteristic, therefore, the choice of solvent is difficult in order to obtain complete recovery (Garcia-Parilla et al., 2009). The ratio of organic/aqueous solvent used for extraction ranged from 0 to $100 \%$ in the method descriptions cited in Table 1. Some of the authors purified the resulting extract with an additional extraction step (Burkhardt et al., 2001, González-Gómez \& Lozano, 2009; Huang \& Mazza, 2011, Homoki et al., 2015) or with SPE (Kirakosyan et al., 2009; Reinholds et al., 2016; Rosado et al., 2017 ), while others did not (Kocadağlı et al., 2014; Islam et al., 2016, Özen and Ekşi, 2016). As the chemistry of plants is very complex, coelution of compounds with similar characteristic may cause false results, therefore the clearing of the extract with a purification step is advantageous. On the other hand, including a further step may cause lower recovery, moreover, it was reported that melatonin can be decomposed by the impurities being present in organic solvents (Feng et al., 2014).

The amino group of serotonin can be protonated, resulting in an increase in the solubility of the molecule in aqueous systems. The $\mathrm{pH}$ values of the aqueous extraction solutions were set to 8.0 or below this value (Table 1.) according to the $\mathrm{pK}$ value of serotonin $(10.4 \pm 0.2$, Chattopadhyay et al., 1996). In the case of inclusion of a purification step with an organic solvent the aqueous extract has 
to be alkalify prior to extraction in order to obtain an acceptable recovery (González-Gómez and Lozano, 2009). For melatonin, the acidic hydrolysis of the amide group is promoted at low $\mathrm{pH}$, if the temperature is elevated, nevertheless, extraction usually occurs at room temperature. Authors, who aimed the simultaneous determination of serotonin and melatonin (GonzálezGómez \& Lozano, 2009; Huang \& Mazza, 2011; Özen and Ekşi, 2016), did not use solutions with acidic $\mathrm{pH}$ during extraction. The parallel extraction of these two indoleamine derivatives from cherry is very scarce in the literature. Huang and Mazza (2011) reported good recovery in methanol extracts for both serotonin (100.9\%) and melatonin (101.3\%). However, ethyl acetate, the purifying solvent, was not appropriate for the extraction of serotonin at all, giving zero recovery for this compound. As a result, the clear-up step with ethyl acetate/water was applied only for melatonin and not for serotonin. The absence of this step might be one of the reasons why the LOQ was more than three orders of magnitude higher for serotonin than for melatonin (Table 1). González-Gómez and Lozano (2009) reported similar LOD values for melatonin and serotonin and their purification step with $0.1 \mathrm{M} \mathrm{KOH} /$ chloroform can be applied for both indoleamine derivatives; nevertheless, the recoveries were lower than in the previous report.

\section{Analytical measurement}

Melatonin is widely distributed in higher plants and plant foods but its concentration is in low ppb range, therefore, its quantification is difficult (Kocadağlı et al., 2014). However, several chromatographic and immunoanalytical methods have been reported to be successful. Immunological techniques include enzyme-linked immunosorbent assay (ELISA), immunoprecipitation and radioimmunoassay (RIA) (Kocadağlı et al., 2014; Garcia-Parrilla et al., 2009; Feng et al., 2014). In the case of RIA, melatonin levels were reported higher compared to results based on GC-MS. A possible explanation was the presence of a compound that cross-reacted with melatonin antiserum (Garcia-Parrilla et al., 2009; Feng et al., 2014). Indoleamine derivatives are thermally labile at the temperatures of gas chromatographic (GC) separation and they are nonvolatile, therefore derivatisation is necessary to improve their stability and volatility. Most often trifluoroacetyl or trimethylsilyl derivatives are analysed using nonpolar stationary phases. Despite the resolution power, and high sensitivity of GC-MS, the use of this technique is scarce for the analysis of melatonin, owing to the 


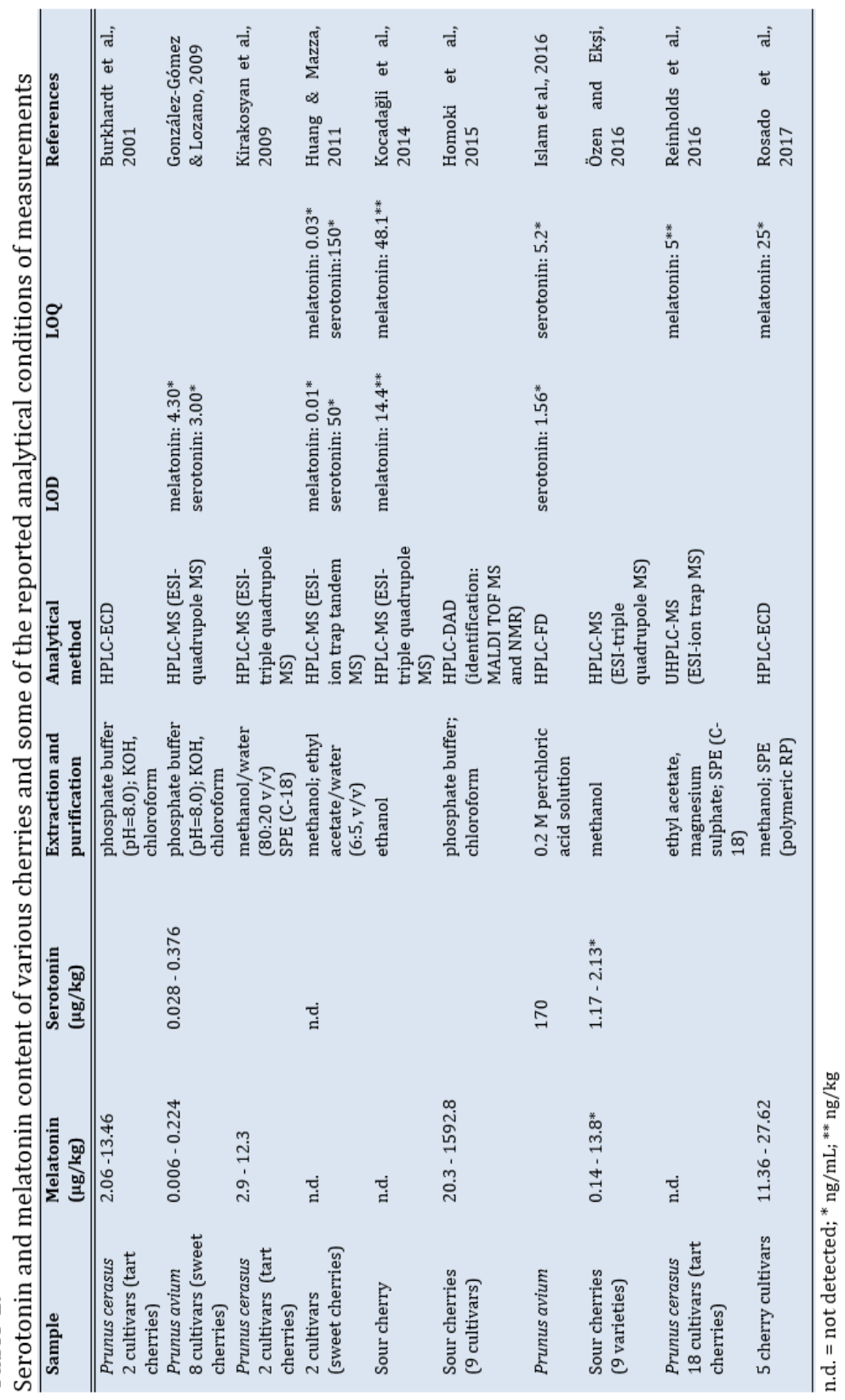


need of derivatization and deuterium-labelled internal standards for accurate quantification (Harumi \& Matsushima, 2000).

The most widespread technique for the analysis of melatonin is highperformance liquid chromatography (HPLC) used in tandem with electrochemical (ECD) or fluorometric detectors (FD) or coupled to mass spectrometer (MS) (Garcia-Parrilla et al., 2009; Kocadağll et al., 2014; Feng et al., 2014). Most of the methods have used reversed phase systems for the separation of compounds, including C18 or C8 columns, and mobile phases containing formate, acetate or phosphate buffer $(\mathrm{pH}=3.4-4.8)$ with acetonitrile or methanol (Burkhardt et al., 2001, Harumi \& Matsushima, 2000; GarciaParrilla et al., 2009; Feng et al., 2014; Homoki et al., 2015; Rosado et al., 2017).

Electrochemical detection is based on the electro-oxidation of the analytes on the electrode surface. The setting of the applied potential highly effects the sensitivity of the detection. In the case of melatonin assay, its maximum value is usually between 700 and 900 V (Harumi \& Matsushima, 2000; Sharma et al., 2018). In general, these sensors are highly sensitive and they have wide linear response. However, reusability and reproducibility can be reduced by the oxidation products which absorb on the surface of the electrode (Sharma et al., 2018). In order to avoid the shortening the lifetime of the electrode the ratio of organic solvent in the mobile phase should be kept below $20-30 \%$ (v/v) (Harumi \&Matsushima, 2000; Feng et al., 2014). This limit may cause difficulties to carry out analysis at a reasonable time in reversed phase gradient elution because the preferable ratio of the strong eluent (methanol or acetonitrile) in order to eluate highly absorbing contaminants at a reasonable time is much higher than this limit value.

Indoleamine derivatives can also be detected with a spectrofluorometer, as indole is highly fluorescent (Bridges \& Williams, 1968). The applied ranges of excitation and emission wavelength are within the ranges of 230-300 $\mathrm{nm}$ and 340-355 nm, respectively (Harumi \& Matsushima, 2000; Feng et al., 2014; Islam et al., 2016). The advantage of FD over ECD is that higher ratio of organic solvents can be used in gradient elution. Contrary, FD is less sensitive than ECD, based on the reported detection limits of melatonin (Harumi \& Matsushima, 2000). The pre-concentration of the sample extract was necessary when melatonin was measured from grape-related foodstuff (Mercolini et al., 2012). In the case of cherry melatonin content was measured with HPLC-MS or with HPLC-ECD and not with HPLC-FD (Table 1).

The melatonin content of different cherry cultivars varied over a wide range (Table 1). Huang and Mazza (2011) did not detect melatonin in sweet 
cherry, while melatonin was measured from other fruits in the range of 0.01$0.1 \mu \mathrm{g} / \mathrm{kg}$. Kocadağli and co-workers (2014), moreover, Reinholds and coworkers (2016) also reported the absence of this indoleamine derivative in cherry, with quantification limits in the range of $0.05-0.005 \mu \mathrm{g} / \mathrm{kg}$.

Analysis of serotonin can be obtained with the same techniques like melatonin, including immunoanalytical and chromatographic methods (Sharma et al., 2018). There is approximately three orders of magnitude difference between the presented values of cherries (Table 1). Islam and coworkers (2016) presented the validation and application of an HPLC-FD method. The serotonin, tryptophan and tryptamine content of various fruits and vegetables, including root and shoot vegetables were determined, melatonin was not measured. They used 5-hydroxy-tryptophane as an internal standard, although this compound is also part of the biosynthetic pathway of serotonin (Feng et al., 2014). In the experiment of González-Gómez and Lozano (2009) the serotonin content of cherry was measured parallel to that of melatonin. The concentrations of the two indoleamines were very similar, being in the sub ppb range. In the assay of Huang and Mazza (2011) serotonin content of sweet cherry was below LOD.

The most important factors which contributed to the observed wide variation in the concentration of indoleamine derivatives were reported as variety and growth condition of cherries (Reinholds et al., 2016), however, there were also large differences in the degree of validation of the analytical methods used for these studies.

\section{Simultaneous analysis of indoleamine derivatives with HPLC-MS}

HPLC-MS has gained popularity for the determination of thermally labile trace compounds being present in complex samples. This fact can partially be explained by the achievement of MS interface technology. The two main parts of an analytical method development are the optimization of the separation and the detection of the compounds. Indoleamine derivatives were separated from trans- and cis-piceid and trans- and cis-resveratrol on a C18 HPLC column using gradient elution with acetonitrile and $0.1 \%(\mathrm{v} / \mathrm{v})$ formic acid, from 5 to $100 \%$, within 40 minutes (Huang \& Mazza, 2011). In the absence of interfering compounds, serotonin and melatonin by themselves can be separated within $15 \mathrm{~min}$, using higher increment of the stronger solvent (González-Gómez \& Lozano, 2009). Parallel determination of serotonin and melatonin was carried out using electrospray ionization (ESI) in HPLC-MS systems. Parameters for ESI can be optimised with the direct introduction of 
standard solution into the ionization source in order to find the optimal capillary temperature and spray voltage for ionization. Spray voltage of 3.50 and $4.00 \mathrm{kV}$ were found as optimal for serotonin and melatonin, respectively, while $250{ }^{\circ} \mathrm{C}$ capillary temperature was the best for both compounds (Huang \& Mazza, 2011). These indoleamines are prone to the formation of positive ions with the addition of a proton to their nitrogen atom; therefore the positive ion mode was used for the formation of quasimolecular ions, m/z 233 for melatonin and m/z 177 for serotonin (González-Gómez \& Lozano, 2009; Huang \& Mazza, 2011). Huang and Mazza (2011) applied tandem MS to detect the fragmentation pathways from precursor ions at m/z 233 and at m/z 177 and quantified their results based on the monitoring of the most abundant fragment ions.

\section{CONCLUSIONS}

Determination of indoleamine derivatives has been started decades ago, however, the primary investigations referred to animal organs, like pineal gland. The analysis of serotonin and melatonin from foods gain actuality following the millennium. In the case of fresh fruits, like sour and sweet cherry, the available literature is limited, despite the fact that the rapid development of HPLC-MS enabled the possibility of the accurate and precise measurement of compounds with low volatility and thermal instability. The reported concentration values cover a wide range, and the factors affecting the serotonin and melatonin levels in cherries have not been completely elucidated. The improvement of selectivity and sensitivity of the analytical methods are key issues to provide reliable data on these trace compounds in foods.

\section{ACKNOWLEDGEMENTS}

This work was supported by project „EFOP-3.6.3-VEKOP-16-2017-00005” cofunded from EU sources and European Structural and Investment Funds and the Hungarian State.

\section{REFERENCES}

Ballistreri, G., Continella, A., Gentile, A., Amenta, M., Fabroni, S. \& Rapisarda, P. (2013). Fruit quality and bioactive compounds relevant to human health of sweet cherry (Prunus avium L.) cultivars grown in Italy. Food Chem., 140, 630-638. DOI: 10.1016/j.foodchem.2012.11.024 
Burkhardt, S., Tan, D.X., Manchester, L.C., Hardeland, R. \& Reiter, R.J. (2001). Detection and quantification of the antioxidant melatonin in Montmorency and Balaton tart cherries (Prunus cerasus). J. Agric. Food Chem., 10, 4898-4902. DOI: 10.1021/jf010321+

Bridges, J.W. \& Williams, R. (1968). The fluorescence of indoles and aniline derivatives. Biochem. J., 107, 225-237. DOI: $10.1042 /$ bj1070225

Chattopadhyay, A., Rukmini, R., Mukherjee, S. (1996). Photophysics of a neurotransmitter: ionization and spectroscopic properties of serotonin. Biophys. J., 71, 1952-1960. DOI: $10.1016 /$ s00063495(96)79393-1

Chockchaisawasdee, S., Golding, J.B., Vuong, Q.V., Papoutsis, K. \&, Stathopoulos C.E. (2016). Sweet cherry: Composition, postharvest preservation, processing and trends for its future use. Trends Food Sci. Tech., 55, 72-83. DOI: 10.1016/j.tifs.2016.07.002

Feng, X., Wang, M., Zhao, Y., Han, P. \& Dai, Y. (2014). Melatonin from different fruit sources, functional roles, and analytical methods. Trends Food Sci. Tech., 37, 21-31. DOI: 10.1016/j.tifs.2014.02.001

Garcia-Parrilla, M. C., Cantos, E. \& Troncoso, A.M. (2009). Analysis of melatonin in foods. Study Review. J. Food Compos. Anal., 22, 177-183. DOI: 10.1016/j.jfca.2008.09.009

González-Gómez, D., Lozano, M., Fernández-León, M.F., Ayuso, M.C., Bernalte, M. J. \& Rodríguez, A.B. (2009). Detection and quantification of melatonin and serotonin in eight Sweet Cherry cultivars (Prunus avium L.). Eur. Food Res. Technol., 229, 223-229. DOI: 10.1007/s00217-009-1042-Z

Harumi, T. \& Matsushima, S. (2000). Separation and assay methods for melatonin and its precursors. Review. J. Chrom. B, 747, 95-110. DOI: 10.1016/s0378-4347(00)00064-5

Homoki, J. R., Gyémánt, Gy., Remenyik J. (2015). Régi hormon új csodája: magyarországi meggyfajták mint természetes melatonin források. Agrártudományi Közlemények, 63, 65-72. WEB: https://dea.lib.unideb.hu/dea/bitstream/handle/2437/235429/FILE_UP_0_acta2015_63_37.pdf ?sequence $=1$ \&isAllowed $=\mathrm{y}$

Huang, X. \& Mazza, G. (2011). Simultaneous analysis of serotonin, melatonin, piceid and resveratrol in fruits using liquid chromatography tandem mass spectrometry. J. Chrom. A, 1218, 3890-3899. DOI: 10.1016/j.chroma.2011.04.049

Islam, J., Shirakawa, H., Nguyen, T.K., Aso, H. \& Komai, M. (2016). Simultaneous analysis of serotonin, tryptophan and tryptamine levels in common fresh fruits and vegetables in Japan using fluorescence HPLC. Food Biosci., 13, 56-59. DOI: 10.1016/j.fbio.2015.12.006

Kirakosyan, A., Seymour, E.M., Llanes, D.E.U., Kaufman, P.B. \& Bolling, S.F. (2009). Chemical profile and antioxidant capacities of tart cherry products. Food Chem., 115, 20-25. DOI: 10.1016/j.foodchem.2008.11.042

Kocadağlı, T., Yılmaz, C. \& Gökmen, V. (2014). Determination of melatonin and its isomer in foods by liquid chromatography tandem mass spectrometry. Food Chem., 153, 151-156. DOI: 10.1016/j.foodchem.2013.12.036

Mercolini, L., Mandrioli, R. \& Raggi, M.A. (2012). Content of melatonin and other antioxidants in graperelated foodstuffs: measurement using a MEPS-HPLC-F method. J. Pineal Res., 53, 21-28. DOI: $\underline{10.1111 / j .1600-079 x .2011 .00967 . x}$

Nawirska-Olszańska, A., Kolniak-Ostek, J., Oziembłowski, M., Ticha, A., Hyšpler, R., Zadak, Z., Židová, P. \& Paprstein, F. (2017). Comparison of old cherry cultivars grown in Czech Republic by chemical composition and bioactive compounds. Food Chem., 228, 136-142. DOI: $\underline{10.1016 / j \text {.foodchem.2017.01.154 }}$

Özen, İ. T., Ekşi, A. (2016). Melatonin and serotonin content of the main sour cherry varieties and commercially produced sour cherry concentrates. European International Journal of Science and Technology, 5, 57-64. WEB: https://www.eijst.org.uk/images/frontImages/gallery/Vol._5_No._4/7._5764.pdf 
Reinholds, I., Pugajeva, I., Radenkovs, V., Rjabova, J., Bartkevics, V. (2016). Development and validation of new ultra-high performance liquid chromatography-hybrid quadrupole-Orbitrap mass spectrometry method for determination of melatonin in fruits. J. Chrom. Sci., 54, 977-984. DOI: 10.1093/chromsci/bmw030

Rosado, T., Henriques, I., Gallardo, E., Duarte, A. P. (2017). Determination of melatonin levels in different cherry cultivars by high-performance liquid chromatography coupled to electrochemical detection. Eur. Food Res. Technol., 243, 1749-1757. DOI: $\underline{10.1007 / \text { s00217-017-2880-8 }}$

Sharma, S., Singh, N., Tomar, V. \& Chandra, R. (2018). A review on electrochemical detection of serotonin based on surface modified electrodes. Biosens. Bioelectron., 107, 76-93. DOI: 10.1016/j.bios.2018.02.013

Wani, A.A., Singh, P., Gul, K., Wani, M.H. \& Langowski, H.C. (2014). Sweet cherry (Prunus avium): Critical factors affecting the composition and shelf life. Food Packaging and Shelf Life, 5, 86-99. DOI: 10.1016/i.fpsl.2014.01.005 